\title{
ON THE IMPORTANCE OF CROSS-SECTIONAL DISTORTIONS AND THEIR INCLUSION IN AN EFFICIENT GBT-FORMULATION
}

\author{
Stephan KUGLER ${ }^{1}$, Peter A. FOTIU ${ }^{1}$ AND Justin MURIN ${ }^{2}$ \\ ${ }^{1}$ Department of Applied and Numerical Mechanics, University of Applied Sciences Wiener Neustadt, \\ Johannes Gutenberg-Straße 3, 2700 Wiener Neustadt, Austria, \\ kugler@fhwn.ac.at, fotiu@fhwn.ac.at, www.fhwn.ac.at \\ 2 Department of Applied Mechanics and Mechatronic of IAMT, Slovak University of Technology in \\ Bratislava, \\ Ilkovičova 3, 81219 Bratislava, Slovakia, \\ justin.murin@stuba.sk,www.stuba.sk
}

Key words: Warping, Distortions, Generalized Beam Theory, Prismatic Beam Structures

\begin{abstract}
This proceeding proposes an efficient generalized beam theory formulation, which accounts for cross-sectional deformations in slender prismatic structures. It was shown by the authors in a recent publication [1] that in-plane distortional deformations and accompanied out of plane warping deformations of the cross-section influence the accuracy of results in beam dynamics especially if thin-walled cross-sections are applied. The GBT formulation proposed in [1] overcomes the inaccuracies of classical beam mechanics, however, requires a two-dimensional plane discretization of the cross-section. The computational complexity can be reduced vastly, if the thin-walled cross-sections can be discretized with one-dimensional elements. Consequently, this proceeding discusses a corresponding derivation, where the line mesh which discretizes the cross-section has six degrees of freedom at each node. The membrane part consists of mass-less micro-polar rotations (drilling rotations) and can be derived independently from the bending part, where a shear elastic formulation is selected.
\end{abstract}

\section{Introduction}

The mechanical properties of slender prismatic homogeneous or inhomogeneous structures made of Functionally Graded Materials (FGM), referred to as beams, are typically analyzed based on Classical Beam Mechanics (CBM). There, it is assumed that the cross-section moves rigidly in space, while out of plane (warping) deformations are only related to non-uniform warping torsion. Any in-plane deformations of the cross-section are neglected. The main advantages of such an approach are without any claim to completeness

- the minimal number of degrees of freedom (three displacements and three rotations of the crosssection added by a single degree of freedom quantifying warping deformations due to non-uniform torsion),

- less modeling time due to simple modeling of supports and joints,

- the possibility of analytic analyses in connection with parametric results without multiple models. 

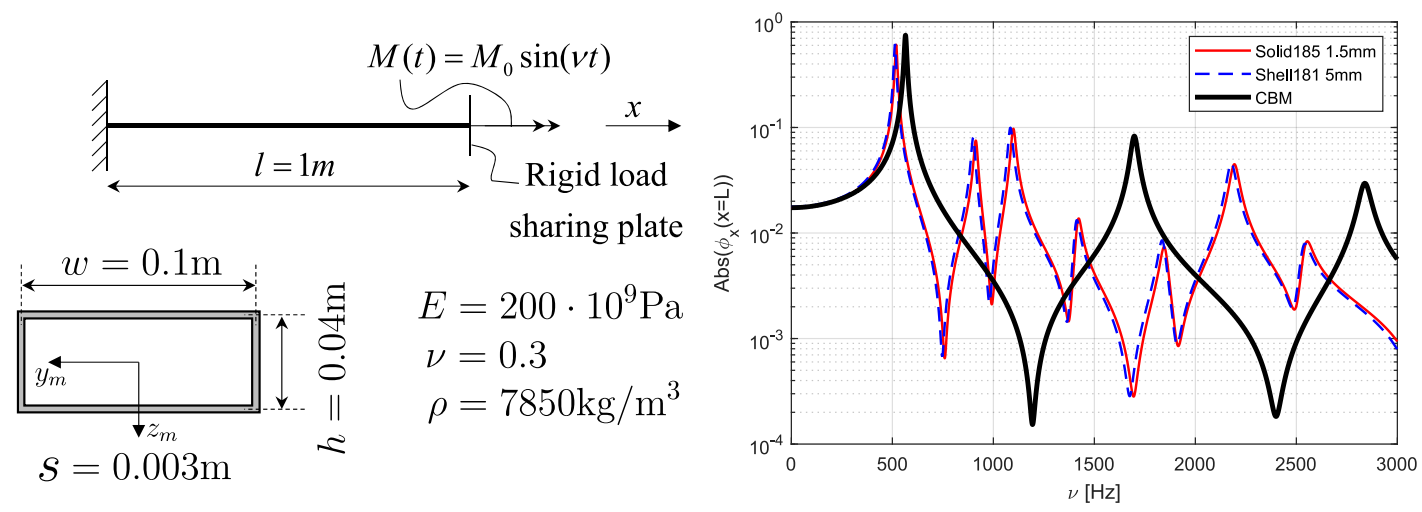

Figure 1: Deficiencies of CBM in analyzing torsional vibrations in shafts made of thin-walled box-type crosssections

In CBM most stiffness quantities related to constitutive parameters and to the cross-sectional shape can be evaluated analytically by simple integration even in case of FGMs where additional homogenization procedures are introduced (see e.g. Murin et al. [2]). However, this does not hold if a consistent evaluation of shear correction factors (required in Timosheko's shear elastic beam theory) is desired, and if stiffness quantities for non-uniform warping torsion (depending on the warping field) are needed. In the former case the procedures by Gruttmann et al. [3] can be applied while in the latter the solution of the Laplace equation has to b cross-sectional shapes have to be applied. Besic lyzed analytically (or very continuum mechanics is significantly smaller com
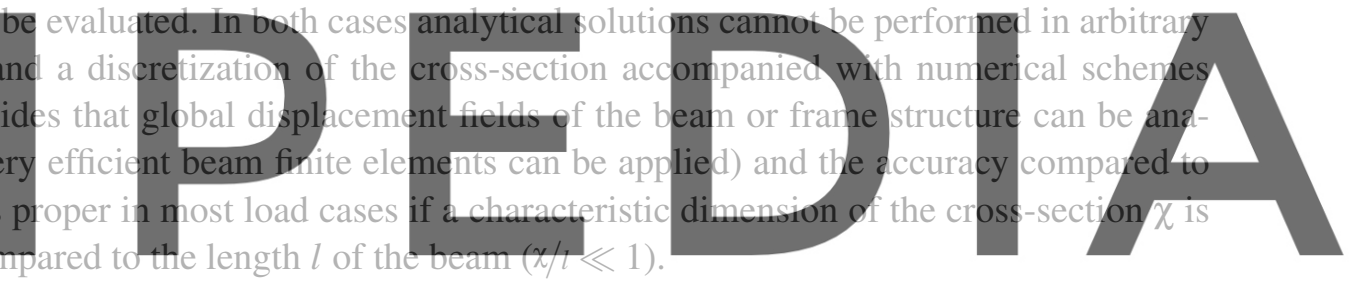

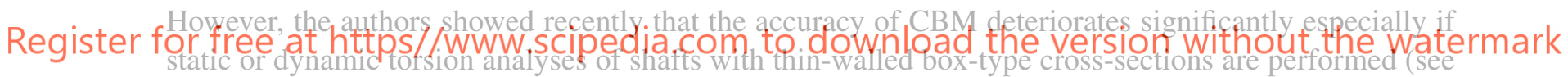

Fig. 1 where torsional vibration is studied in a quite slender shaft, indicating that CBM does not even come close to continuum (or shell) results since in-plane distortions influence the mechanical properties significantly $\left.[1,4]^{1}\right)$. These effects are also observed by Sapountzakis et al. $[5,6]$ where also buckling analyses are considered. In order to circumvent the insufficient accuracy of CBM in thin-walled single or multi-cell box type cross-sections, however, maintaining a moderate number of global degrees of freedom, a Generalized Beam Theory (GBT) can be applied (see [1] and [5] for a comprehensive literature review and a discussion of main contributions in that field). After all, GBT is a two-step algorithm consisting of a cross-sectional analysis defining warping and distortional fields, followed by a member analysis, where those deformation fields are weighed axially along the beam. Kugler et al. propose a new form of GBT and the main ingredients are summarized subsequently (for additional details refer to $[1,4])$ :

1. Cross-sectional analysis: The cross-section is discretized with Semi-Analytical Finite Elements

\footnotetext{
${ }^{1}$ See $[1,4]$ where additional deficiencies of CBM are discussed in case of unsymmetrical cross-sections and FGM crosssections.
} 
(SAFE) [7] to efficiently analyze a Reference Beam Problem (RBP) with arbitrary length $l_{R B}$ continuum-mechanically. There, the displacement fields within the RBP are interpolated based on finite element type shape functions with respect to the cross-sectional coordinates $y_{m}$ and $z_{m}$, while their axial variation is modeled based on a Fourier series. The specific form of the Fourier series expansion defines the kinematic boundary conditions of the RBP at $x=0$ and $x=l_{R B}$, and we apply a variation of the transverse displacements based on sine functions and the variation of the axial displacements based on cosine functions ${ }^{2}$. Thus, we have on element level,

$$
\left[\begin{array}{c}
u_{x}(x, \xi, \eta) \\
u_{y_{m}}(x, \xi, \eta) \\
u_{z_{m}}(x, \xi, \eta)
\end{array}\right]=\sum_{n}\left[\begin{array}{ccc}
\mathbf{N} \cos \left(\alpha_{n} x\right) & \mathbf{0} & \mathbf{0} \\
\mathbf{0} & \mathbf{N} \sin \left(\alpha_{n} x\right) & \mathbf{0} \\
\mathbf{0} & \mathbf{0} & \mathbf{N} \sin \left(\alpha_{n} x\right)
\end{array}\right]\left[\begin{array}{c}
\mathbf{U}_{x}^{(e) n} \\
\mathbf{U}_{y_{m}}^{(e) n} \\
\mathbf{U}_{z_{m}}^{(e) n}
\end{array}\right]
$$

with the bilinear shape functions $\mathbf{N}(\xi, \eta)$ with finite element parameter coordinates $\xi$ and $\eta$, and the nodal displacement amplitudes $\mathbf{U}_{i}^{(e) n}=\left[\begin{array}{lll}U_{i}^{(1) n} & \ldots & U_{i}^{(4) n}\end{array}\right]^{T}$ depending on the number of harmonic $n$, while $\alpha_{n}=\frac{n \pi}{l_{P B}}$. Now, the key idea of our proposed procedure is the application of only the FIRST wave number $n=1$ and use (1) to perform a free vibration analysis (modal analysis in connection with the principle of virtual work, three-dimensional strain-displacement relations and three-dimensional elasticity) of the RBP. Each resulting eigenvector $\Gamma_{i}$ ( $i$-th mode shapes) in ascending order of corresponding eigenvalues (resonance frequencies $\omega_{i}$ of the RBP) is split up into couples consisting of out of plane warping modes,
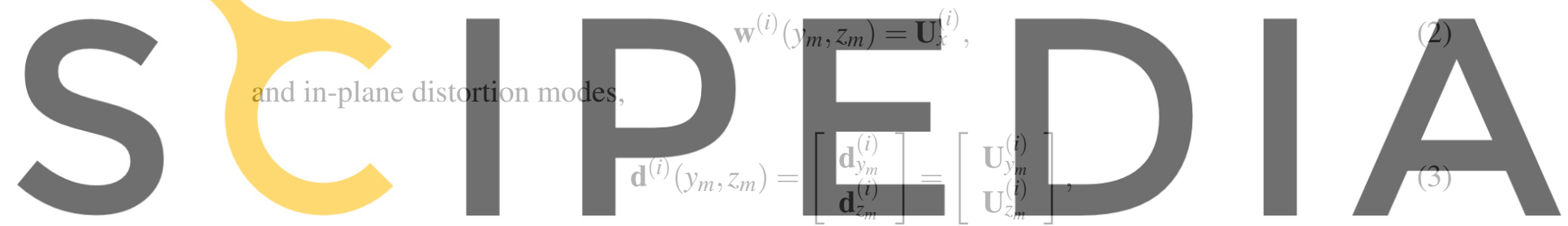

which are scaled independently in order to avoid small numbers. The first four modes $i=1 \ldots 4$

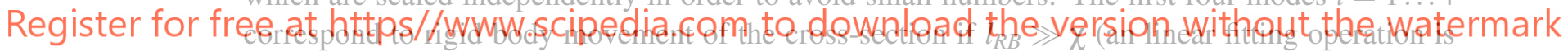

proposed to extract the pure rigid body modes) and all mode couples $i>4$ contribute to a higher order beam theory. These $i=1 \ldots i_{\max }$ mode couples with $i_{\max }>4$ represent pairs of warping and distortion modes of the cross-section and are weighed axially in the up-coming member analysis.

2. Member analysis: The true beam problem (opposing to the afore-mentioned artificial reference beam problem) is discretized with four noded GBT beam elements (cubic finite element type shape functions to avoid locking phenomena) and the displacement field in each element results from weighing the warping and distortion mode couples (2) and (3) axially,

$$
\begin{gathered}
u_{x}(\xi, \eta, x)=\sum_{i=1}^{i_{\max }} u_{x}^{(i)}(\xi, \eta, x)=\sum_{i=1}^{i_{\max }} \mathbf{N}(\xi, \eta) \mathbf{w}_{e}^{(i)} \alpha_{i}(x), \\
u_{y_{m}}(\xi, \eta, x)=\sum_{i=1}^{i_{\max }} u_{y_{m}}^{(i)}(\xi, \eta, x)=\sum_{i=1}^{i_{\max }} \mathbf{N}(\xi, \eta) \mathbf{d}_{y_{m} e}^{(i)} \beta_{i}(x),
\end{gathered}
$$

\footnotetext{
${ }^{2}$ This leads to a simply supported RBP for transverse action, a double sided fork supported RBP for torsional action and an unrestrained RBP for axial action (see [1] for a thorough discussion).
} 


$$
u_{z_{m}}(\xi, \eta, x)=\sum_{i=1}^{i_{\max }} u_{z_{m}}^{(i)}(\xi, \eta, x)=\sum_{i=1}^{i_{\max }} \mathbf{N}(\xi, \eta) \mathbf{d}_{z_{m}}^{(i)} \beta_{i}(x) .
$$

The GBT degrees of freedom $\alpha_{i}(x)$ and $\beta_{i}(x)$ are interpolated based on cubic shape functions,

$$
\alpha_{i}(x)=\left[\begin{array}{lll}
N_{B 1} & \cdots & N_{B 4}
\end{array}\right]\left[\begin{array}{c}
A_{1}^{(i)} \\
\vdots \\
A_{4}^{(i)}
\end{array}\right], \beta_{i}(x)=\left[\begin{array}{lll}
N_{B 1} & \cdots & N_{B 4}
\end{array}\right]\left[\begin{array}{c}
B_{1}^{(i)} \\
\vdots \\
B_{4}^{(i)}
\end{array}\right]
$$

and the displacements (4)-(6) are introduced into the principle of virtual work where strains $\varepsilon$ and stresses $\sigma$ are found using the corresponding three-dimensional definition,

$$
\varepsilon_{k l}=\frac{1}{2}\left(u_{k, l}+u_{l, k}\right), \sigma_{m n}=c_{m n k l} \varepsilon_{k l} .
$$

There, a comma in the index refers to partial derivative and $c_{m n k l}$ denotes the elasticity tensor, where we assume one-dimensional elasticity for each stress component. A straight forward derivation detailed in [1] leads to GBT beam element stiffness and mass matrices, which are assembled to the global problem definition of the true beam problem. Physical convergence of the GBT results compared to continuum results is ensured by the number of contributing warping and distortion couples $i_{\max }$, while numerical convergence occurs due to the number of semi-analytical finite elements in the cross-sectional analysis and due to the number of GBT beam elements in the member

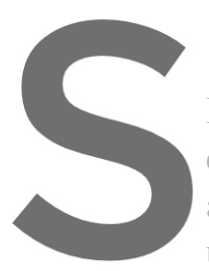
analysis.

It is shown in $[1,4]$ that th

especially in cases where the

applied. Additionally,

unsymmetric cross-section
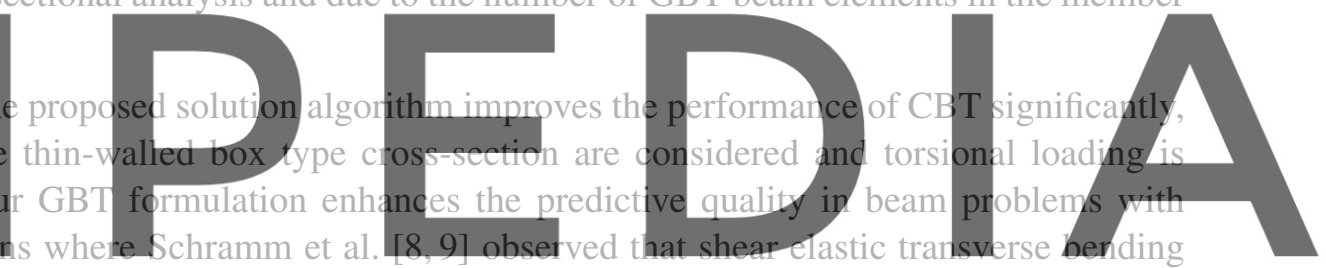

load cases do not decouple based on the eigenvector of the bending stiffness tensor. Further, it can be

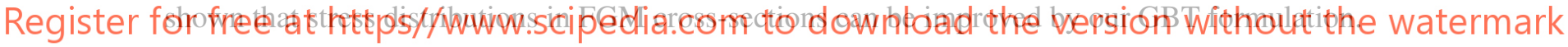

The required computational effort in our GBT formulation is significantly lower compared to three-

dimensional continuum formulations: The cross-section has to be discretized with plane elements (and three degrees of freedom at each node (1)) in the cross-sectional analysis, while coarse GBT beam element discretizations suffice in the member analysis due to the cubic shape functions in connection with $2 i_{\max }$ nodal degrees of freedom. Note that the computational complexity is mostly defined by the number of plane elements in the cross-sectional analysis. Firstly, the cost of the eigenvalue/vector decomposition of the RBP with $3 n$ degrees of freedom is roughly proportional to $(3 n)^{3}$, secondly in the member analysis products of large matrices of the type $\mathbf{P}^{T} \mathbf{A P}$ have to be carried out $i_{\max }^{2}$ times, where $\mathbf{A}$ is a $6 n \times 6 n$ matrix and $\mathbf{P}$ is a $6 n \times 6$ projection matrix in order to couple the mode pairs from the cross-sectional analysis.

From the above analysis of computational complexity and from the observation that the increase of accuracy of our GBT formulation compared to CBT is most prominent in thin-walled cross-sections of wall thickness $s$, we establish the wish for improvement which will be discussed in this procedure: The continuum mechanics of the RBP with a thin-walled cross-section using SAFE requires at least two elements in wall-thickness direction and it is well known that the aspect ratio of continuum elements is 
aimed to be one. This means that the flange and web lengths $l_{i}$ of the thin-walled cross-section have to be discretized with $2 l_{i} / s$ elements leading to large numbers of nodes $n$ within our GBT approach. Things can be improved considerably if line meshes can be used to discretize the cross-section. Then, the gain in efficiency is similar to the application of shell elements in thin-walled structures compared to an analysis using three-dimensional continuum elements for those structures, which is known to be enormous. However, this requires a semi-analytical finite SHELL elements (SAFSE) approach within the RBP (see Sect. 2), which - at least to the author's knowledge - has never been proposed in literature. While the classical SAFE approach introduces three degrees of freedom at each node (1) the SAFSE strategy requires six degrees of freedom (three displacements and three rotations) at each node. From shell theory it is well known that only five degrees of freedom are present in a classical approach (three displacements and two bending angles) and the sixth in-plane rotational degree of freedom - frequently referred to as drilling rotation - is missing. Much literature is available for the introduction of drilling rotations in membrane elements (unphysical approaches, geometrical approaches using Allman rotations $[10]$, strategies using enhanced functionals $[11], \ldots$ ), however, the authors recently proposed the inclusion of drilling rotations based on a micro-polar theory of elasticity, where rotations of a point occur naturally [12-16]. These ideas will be incorporated here in the membrane part of SAFSE while shear elastic formulations will be used in the bending part of the formulation. A coupling of membrane and bending part is not required (and membrane locking is therefore circumvented by principle), if the line mesh of the semi-analytical finite shell element is straight. Transverse shear locking is circumvented by using cubic shape functions, while the principal ideas of the GBT formulation originated in [1,4] and summarized above remain unchanged.
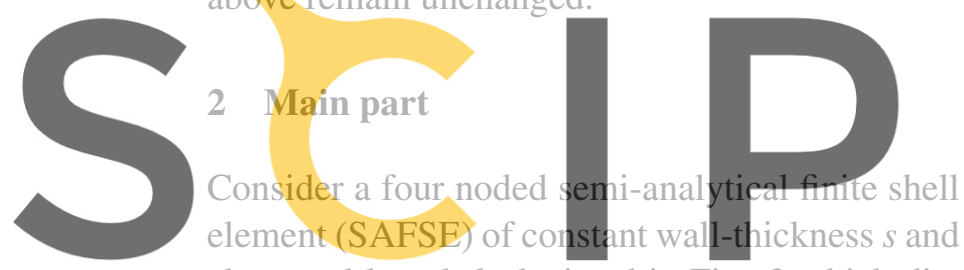

elemental length $l_{e}$ depicted in Fig. 2 which dis-
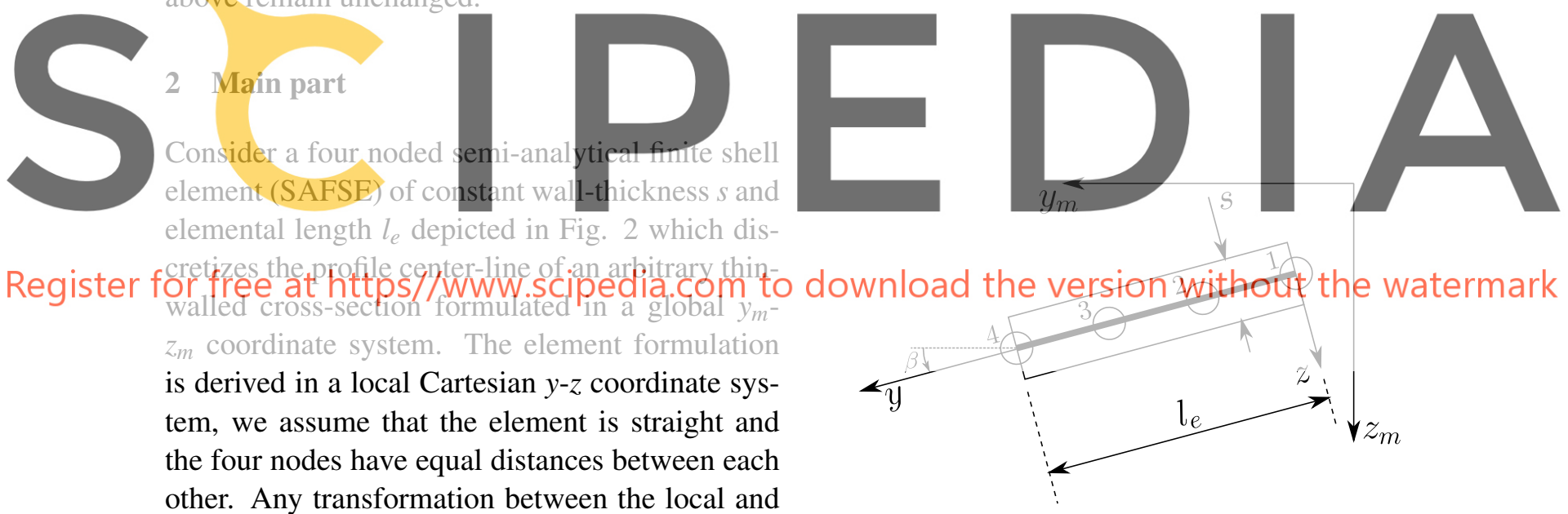

Figure 2: Four noded semi-analytical finite shell element (SAFSE) using a orthogonal rotation matrix $\mathbf{R}$,

$$
\left[\begin{array}{l}
a_{y_{m}} \\
a_{z_{m}}
\end{array}\right]=\left[\begin{array}{cc}
\cos \beta & -\sin \beta \\
\sin \beta & \cos \beta
\end{array}\right]\left[\begin{array}{l}
a_{y} \\
a_{z}
\end{array}\right],
$$

where we assume $-\pi \leq \beta \leq \pi$ and $\mathbf{a}$ denotes any vector. In the first step (cross-sectional analysis) a reference beam problem (RBP) of length $l_{R B}$ has the arbitrary thin-walled cross-section which is discretized using the SAFSEs $\left(x=x_{m}\right)$. Each node has three displacement and three rotational degrees of freedom, $u_{i}$ and $\varphi_{i}$ with $i=x, y, z$, which can be grouped into membrane and bending degrees of freedom and the derivation of the corresponding part of the stiffness matrix can be carried out independently as long 
as the SAFSE is straight. In order to derive the cross-sectional analysis based on the RBP introduced in $[1,4]$ (see footnote 2 ), the the analytical variations in axial direction are proportional to sine or cosine functions, and we have,

$$
\text { membrane }\left\{\begin{array} { r l } 
{ u _ { x } } & { \sim \operatorname { c o s } \alpha x } \\
{ u _ { y } } & { \sim \operatorname { s i n } \alpha x } \\
{ \varphi _ { z } } & { \sim \operatorname { c o s } \alpha x }
\end{array} \text { , bending } \left\{\begin{array}{l}
u_{z} \sim \sin \alpha x \\
\varphi_{x} \sim \sin \alpha x \\
\varphi_{y} \sim \cos \alpha x
\end{array}\right.\right.
$$

with $\alpha=\pi / l_{R B}$. In contrast the variations of those degrees of freedom in $y$-direction are based on cubic finite element type shape functions $N_{i}(y)$ with $i=1 \ldots 4$.

The RBP's membrane part is modeled due to the virtual work of inner forces,

$$
\int \delta \mathbf{u}_{m}^{T} \rho \mathbf{a}_{m} d V+\int \delta \varepsilon_{s m}^{T} \mathbf{C} \varepsilon_{s m} d V+2 \mu \int \delta \varepsilon_{a m} \varepsilon_{a m} d V=0
$$

where the first term models inertia with density $\rho$, acceleration $\mathbf{a}_{m}=\ddot{\mathbf{u}}_{m}=\partial^{2} / \partial t^{2} \mathbf{u}_{m}$ and $\mathbf{u}_{m}=\left[\begin{array}{cc}u_{x m} & u_{y m}\end{array}\right]^{T}$ denoting membrane displacements. A variation symbol is depicted as $\delta$. The second part in (11) refers to the classical symmetric membrane strain tensor $\varepsilon_{s}=\left[\begin{array}{lll}\varepsilon_{x x}^{s} & \varepsilon_{y y}^{s} & \gamma_{x y}^{s}\end{array}\right]^{T}$ with

$$
\varepsilon_{x x}^{s}=u_{x, x}, \varepsilon_{y y}^{s}=u_{y, y}, \gamma_{x y}^{s}=u_{x, y}+u_{y, x}
$$

where a comma in the index denotes a partial derivative. The elasticity matrix $\mathbf{C}$ is the plane stress elasticity matrix,

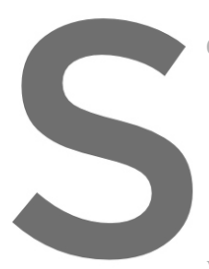

where $E$ denotes the Young's modulus, $v$ the Poiss
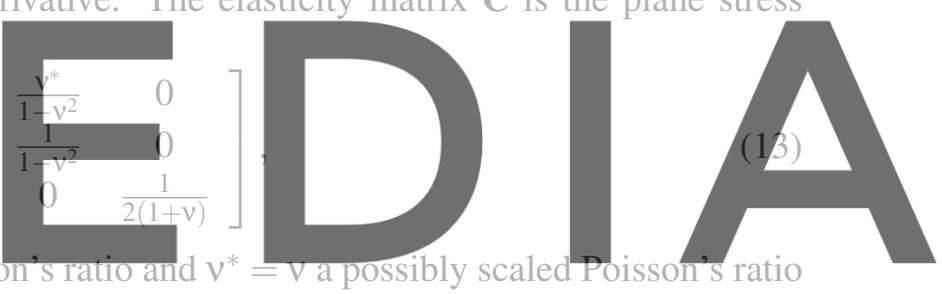

defining the coupling between normal stresses and normal strains. The third part in (11) stems from the

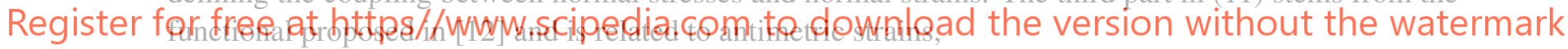

$$
\varepsilon_{a}=\frac{1}{2}\left(u_{x, y}-u_{y, x}\right)+\varphi_{z}
$$

while $\mu$ denotes a constitutive parameter which is related to the possibly scaled local shear modulus $\mu=\varkappa^{E} /(2(1+v))$. The assumed displacement field then reads,

$$
\left[\begin{array}{l}
u_{x m} \\
u_{y m}
\end{array}\right]=\left(\left[\begin{array}{ccc}
\mathbf{0} & \mathbf{0} & \mathbf{0} \\
\mathbf{0} & \mathbf{N} & \mathbf{0}
\end{array}\right] \sin (\alpha x)+\left[\begin{array}{ccc}
\mathbf{N} & \mathbf{0} & \mathbf{0} \\
\mathbf{0} & \mathbf{0} & \mathbf{0}
\end{array}\right] \cos (\alpha x)\right)\left[\begin{array}{c}
\mathbf{U}_{x} \\
\mathbf{U}_{y} \\
\Phi_{z}
\end{array}\right]
$$

with $\mathbf{N}=\left[\begin{array}{lll}N_{1}(y) & \cdots & N_{4}(y)\end{array}\right]$ and $\alpha=\pi / l_{R B}$, the symmetric strain tensor is given by,

$$
\left[\begin{array}{c}
\varepsilon_{x x}^{s m} \\
\varepsilon_{y y}^{s m} \\
\gamma_{x y}^{s m}
\end{array}\right]=\left(\left[\begin{array}{ccc}
-\alpha \mathbf{N} & \mathbf{0} & \mathbf{0} \\
\mathbf{0} & \mathbf{N}_{, y} & \mathbf{0} \\
\mathbf{0} & \mathbf{0} & \mathbf{0}
\end{array}\right] \sin (\alpha x)+\left[\begin{array}{ccc}
\mathbf{0} & \mathbf{0} & \mathbf{0} \\
\mathbf{0} & \mathbf{0} & \mathbf{0} \\
\mathbf{N}_{, y} & \alpha \mathbf{N} & \mathbf{0}
\end{array}\right] \cos (\alpha x)\right)\left[\begin{array}{c}
\mathbf{U}_{x} \\
\mathbf{U}_{y} \\
\Phi_{z}
\end{array}\right],
$$


and the antimetric strain component is

$$
\varepsilon_{a m}=\left[\begin{array}{lll}
\frac{1}{2} \mathbf{N}_{, y} & -\frac{1}{2} \alpha \mathbf{N} & \mathbf{N}
\end{array}\right] \cos (\alpha x)\left[\begin{array}{l}
\mathbf{U}_{x} \\
\mathbf{U}_{y} \\
\Phi_{z}
\end{array}\right] .
$$

In (15)-(17) the elemental nodal degree of freedom vector for membrane action $\mathbf{d}_{m e}=\left[\begin{array}{lll}\mathbf{U}_{x}^{T} & \mathbf{U}_{y}^{T} & \Phi_{z}^{T}\end{array}\right]^{T}$ consists of the nodal values $\mathbf{U}_{i}^{T}=\left[\begin{array}{lll}U_{i 1} & \cdots & U_{i 4}\end{array}\right]$ with $i=x, y$ and $\Phi_{z}^{T}=\left[\begin{array}{lll}\Phi_{z 1} & \cdots & \Phi_{z 4}\end{array}\right]$ and in connection with (11) we find on element level

$$
\mathbf{M}_{m e} \ddot{\mathbf{d}}_{m e}+\mathbf{K}_{m e} \mathbf{d}_{m e}=\mathbf{0} .
$$

There, it has to be pointed out that the volume integration $d V=s d x d y$ can be carried out analytically, and we observe that no rotary inertia is assigned to the drilling rotations $\Phi_{z}$ leading to a positive semi-definite mass matrix.

The RBP's shear elastic bending part is derived based on the functional [13,14],

$$
\int \delta \mathbf{u}_{b}^{T} \rho \mathbf{a}_{b} d V+\int \delta \varepsilon_{s b}^{T} \mathbf{C} \varepsilon_{s b} d V+\frac{E \alpha_{s}}{2(1+v)} \int \delta \bar{\gamma} \bar{\gamma} d V=0,
$$

with $\mathbf{a}_{b}$ denoting the second time derivative of bending displacements $\mathbf{u}_{b}=\left[\begin{array}{lll}u_{x b} & u_{y b} & u_{z b}\end{array}\right]^{T}, \varepsilon_{s b}=$

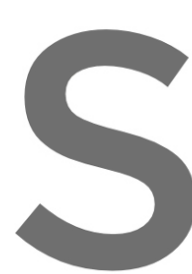
$\left[\begin{array}{lll}\varepsilon_{x x}^{b} & \varepsilon_{y y}^{b} & \gamma_{x y}^{b}\end{array}\right]^{T}$ refento elasticity matrix from ( third summand in (19) known mismatch between homogeneous cases we as
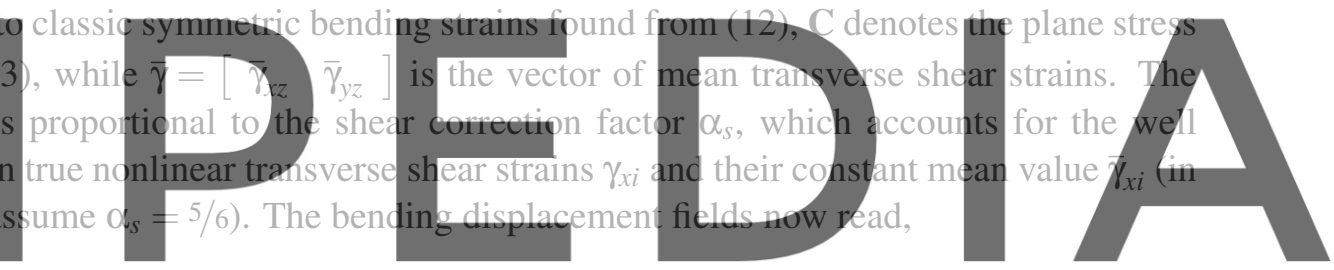

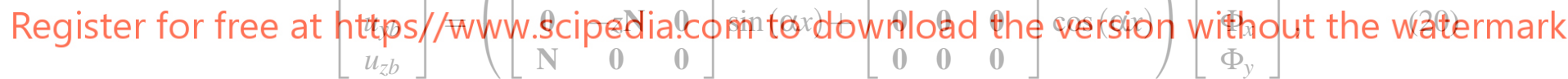

the bending strain tensor is

$$
\left[\begin{array}{c}
\varepsilon_{x x}^{s b} \\
\varepsilon_{y y}^{s b} \\
\gamma_{x y}^{s b}
\end{array}\right]=\left(\left[\begin{array}{ccc}
\mathbf{0} & \mathbf{0} & -z \alpha \mathbf{N} \\
\mathbf{0} & -z \mathbf{N}_{y} & \mathbf{0} \\
\mathbf{0} & \mathbf{0} & \mathbf{0}
\end{array}\right] \sin (\alpha x)+\left[\begin{array}{ccc}
\mathbf{0} & \mathbf{0} & \mathbf{0} \\
\mathbf{0} & \mathbf{0} & \mathbf{0} \\
\mathbf{0} & -z \alpha \mathbf{N} & z \mathbf{N}_{, y}
\end{array}\right] \cos (\alpha x)\right)\left[\begin{array}{l}
\mathbf{U}_{z} \\
\Phi_{x} \\
\Phi_{y}
\end{array}\right]
$$

and the transverse shear strains are found from $\bar{\gamma}_{x z}=u_{x b, z}+u_{z b, x}$ and $\bar{\gamma}_{y z}=u_{y b, z}+u_{z b, y}$ as,

$$
\left[\begin{array}{c}
\bar{\gamma}_{x z} \\
\bar{\gamma}_{y z}
\end{array}\right]=\left(\left[\begin{array}{ccc}
\mathbf{0} & \mathbf{0} & \mathbf{0} \\
\mathbf{N}_{, y} & -\mathbf{N} & \mathbf{0}
\end{array}\right] \sin (\alpha x)+\left[\begin{array}{ccc}
\alpha \mathbf{N} & \mathbf{0} & \mathbf{N} \\
\mathbf{0} & \mathbf{0} & \mathbf{0}
\end{array}\right] \cos (\alpha x)\right)\left[\begin{array}{c}
\mathbf{U}_{z} \\
\Phi_{x} \\
\Phi_{y}
\end{array}\right]
$$

Using the interpolations (20)-(22) in connection with the virtual work (19) leads to the finite element equation for the bending part

$$
\mathbf{M}_{b e} \ddot{\mathbf{d}}_{b e}+\mathbf{K}_{b e} \mathbf{d}_{b e}=\mathbf{0},
$$


with $\mathbf{d}_{b e}=\left[\begin{array}{lll}\mathbf{U}_{z}^{T} & \Phi_{x}^{T} & \Phi_{y}^{T}\end{array}\right]^{T}$ and the nodal values $\mathbf{U}_{z}^{T}=\left[\begin{array}{lll}U_{z 1} & \cdots & U_{z 4}\end{array}\right]$ and $\Phi_{i}^{T}=\left[\begin{array}{lll}\Phi_{i 1} & \cdots & \Phi_{i 4}\end{array}\right]$ with $i=x, y$. Again, all integrations can be carried out analytically and we note that rotary inertia is assigned to the bending angles leading to a positive definite mass matrix for bending $\mathbf{M}_{b e}$.

The RBP's semi-analytical finite shell element (SAFSE) is found easily by assembling the membrane and bending part,

$$
\left[\begin{array}{cc}
\mathbf{M}_{m e} & \mathbf{0} \\
\mathbf{0} & \mathbf{M}_{b e}
\end{array}\right]\left[\begin{array}{c}
\ddot{\mathbf{d}}_{m e} \\
\ddot{\mathbf{d}}_{b e}
\end{array}\right]+\left[\begin{array}{cc}
\mathbf{K}_{m e} & \mathbf{0} \\
\mathbf{0} & \mathbf{K}_{b e}
\end{array}\right]\left[\begin{array}{c}
\mathbf{d}_{m e} \\
\mathbf{d}_{b e}
\end{array}\right]=\mathbf{0}
$$

a classical assembly procedure from elemental to global matrices is understood after transforming the matrices to the global member coordinate system $y_{m}-z_{m}$ using (9),

$$
\mathbf{M d}+\mathbf{K d}=\mathbf{0},
$$

and an eigenvalue problem is set up based on the Ansatz $\mathbf{d}=\widehat{\mathbf{d}} \sin \omega t$. The eigenvalues $\omega_{i}$ are used to order the eigenvectors $\widehat{d}_{i}$ ascendingly, and the cross-sectional analysis is according to $[1,4]$ completed by extracting from each eigenvector $i$ the nodal warping and distortional modes $\mathbf{w}_{i}=\left[\begin{array}{lll}u_{x i} & \varphi_{y i} & \varphi_{z i}\end{array}\right]^{T}$ and $\mathbf{d}_{i}=\left[\begin{array}{lll}u_{y i} & u_{z i} & \varphi_{x i}\end{array}\right]^{T}$, respectively. Independent scaling is only performed based on the displacement components and the first four modes are fitted to true rigid body modes according to [1,4]. These mode couples from $i=1 \ldots i_{\max }$ are the basis for the upcoming member analysis.

The member analysis is derived similarly to the procedures in $[1,4]$, where each warping mode $w_{i}$ and each distortional mode $\mathbf{d}_{i}$ is weighed axially based on a independent degree of freedom, $\alpha_{i}\left(x_{e}\right)$ and

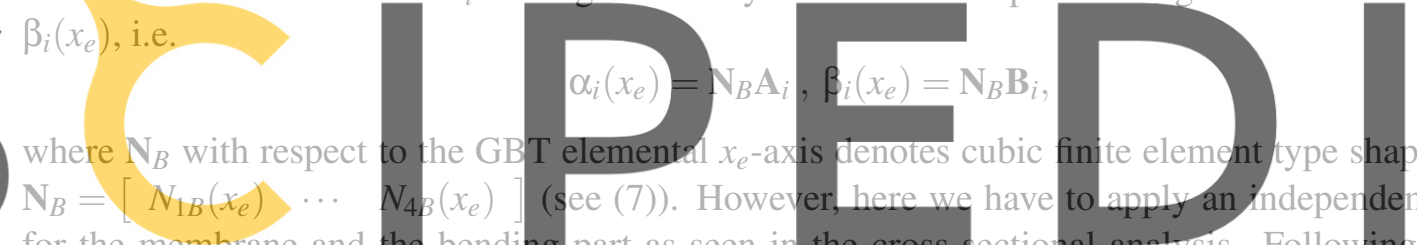

for the membrane and the bending part as seen in th

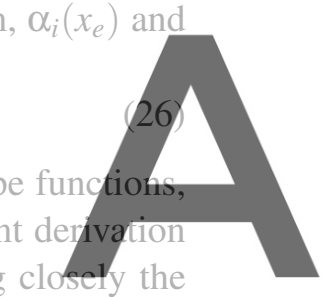

notations in [1] we get for each SAFSE $e$ and each mode couple $i$ membrane and bending displacement

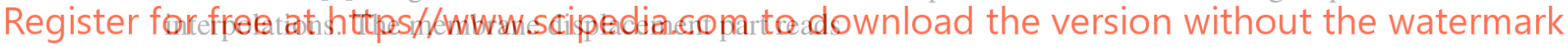

$$
\left[\begin{array}{l}
u_{x e}^{(i) m} \\
u_{y e}^{(i) m}
\end{array}\right]=\mathbf{N}_{3 D}^{m} \mathbf{u}_{u}^{(i) m},
$$

with

$$
\mathbf{N}_{3 D}^{m}=\left[\begin{array}{ccc}
\mathbf{N}(y) & \mathbf{0} & \mathbf{0} \\
\mathbf{0} & \mathbf{N}(y) & \mathbf{0}
\end{array}\right]
$$

and

$$
\begin{gathered}
\mathbf{u}_{u}^{(i) m}=\aleph_{u e}^{(i) m} \mathbf{N}_{B u}^{m} \Xi_{e}^{(i)}= \\
=\left[\begin{array}{ccc}
\mathbf{U}_{x e}^{(i)} & \mathbf{0} & \mathbf{0} \\
\mathbf{0} & \mathbf{U}_{y e}^{(i)} & \mathbf{0} \\
\mathbf{0} & \mathbf{0} & \Phi_{z e}^{(i)}
\end{array}\right]\left[\begin{array}{cc}
\mathbf{N}_{B}\left(x_{e}\right) & \mathbf{0} \\
\mathbf{0} & \mathbf{N}_{B}\left(x_{e}\right) \\
\mathbf{N}_{B}\left(x_{e}\right) & \mathbf{0}
\end{array}\right]\left[\begin{array}{l}
\mathbf{A}_{i} \\
\mathbf{B}_{i}
\end{array}\right],
\end{gathered}
$$


while the bending displacement part is

$$
\left[\begin{array}{c}
u_{x e}^{(i) b} \\
u_{y e}^{(i) b} \\
u_{z e}^{(i) b}
\end{array}\right]=\mathbf{N}_{3 D}^{b} \mathbf{u}_{u}^{(i) b}
$$

with

$$
\mathbf{N}_{3 D}^{b}=\left[\begin{array}{ccc}
\mathbf{0} & \mathbf{0} & z \mathbf{N}(y) \\
\mathbf{0} & -z \mathbf{N}(y) & \mathbf{0} \\
\mathbf{N}(y) & \mathbf{0} & \mathbf{0}
\end{array}\right]
$$

and

$$
\begin{gathered}
\mathbf{u}_{u}^{(i) b}=\aleph_{u e}^{(i) b} \mathbf{N}_{B u}^{b} \Xi_{e}^{(i)}= \\
=\left[\begin{array}{ccc}
\mathbf{U}_{z e}^{(i)} & \mathbf{0} & \mathbf{0} \\
0 & \Phi_{x e}^{(i)} & 0 \\
0 & 0 & \Phi_{y e}^{(i)}
\end{array}\right]\left[\begin{array}{cc}
\mathbf{0} & \mathbf{N}_{B}\left(x_{e}\right) \\
0 & \mathbf{N}_{B}\left(x_{e}\right) \\
\mathbf{N}_{B}\left(x_{e}\right) & 0
\end{array}\right]\left[\begin{array}{l}
\mathbf{A}_{i} \\
\mathbf{B}_{i}
\end{array}\right] .
\end{gathered}
$$

The corresponding strain interpolations for the membrane part are divided into their symmetric and antimetric part, and this results with (12) and (14) into,
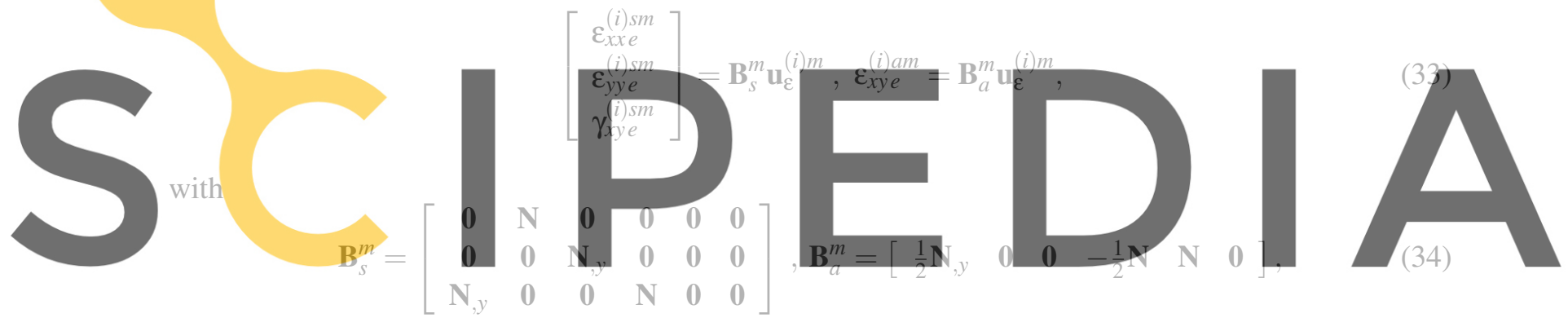

Register fordree at https//www.scipedia.com to download the version without the watermark

$$
\mathbf{u}_{\varepsilon}^{(i) m}=\boldsymbol{\aleph}_{\varepsilon e}^{(i) m} \mathbf{N}_{B \varepsilon}^{m} \Xi_{e}^{(i)}=
$$

$$
=\left[\begin{array}{cccccc}
\mathbf{U}_{x e}^{(i)} & \mathbf{0} & \mathbf{0} & \mathbf{0} & \mathbf{0} & \mathbf{0} \\
\mathbf{0} & \mathbf{U}_{x e}^{(i)} & \mathbf{0} & \mathbf{0} & \mathbf{0} & \mathbf{0} \\
\mathbf{0} & \mathbf{0} & \mathbf{U}_{y e}^{(i)} & \mathbf{0} & \mathbf{0} & \mathbf{0} \\
\mathbf{0} & \mathbf{0} & \mathbf{0} & \mathbf{U}_{y e}^{(i)} & \mathbf{0} & \mathbf{0} \\
\mathbf{0} & \mathbf{0} & \mathbf{0} & \mathbf{0} & \Phi_{z e}^{(i)} & \mathbf{0} \\
\mathbf{0} & \mathbf{0} & \mathbf{0} & \mathbf{0} & \mathbf{0} & \Phi_{z e}^{(i)}
\end{array}\right]\left[\begin{array}{cc}
\mathbf{N}_{B}\left(x_{e}\right) & \mathbf{0} \\
\mathbf{N}_{B}^{\prime}\left(x_{e}\right) & \mathbf{0} \\
\mathbf{0} & \mathbf{N}_{B}\left(x_{e}\right) \\
\mathbf{0} & \mathbf{N}_{B}^{\prime}\left(x_{e}\right) \\
\mathbf{N}_{B}\left(x_{e}\right) & \mathbf{0} \\
\mathbf{N}_{B}^{\prime}\left(x_{e}\right) & \mathbf{0}
\end{array}\right]\left[\begin{array}{c}
\mathbf{A}_{i} \\
\mathbf{B}_{i}
\end{array}\right],
$$

where $\mathbf{N}_{B}^{\prime}$ denotes the derivative of $\mathbf{N}_{B}$ with respect to $x_{e}$. The bending and transverse shear strains are found as,

$$
\left[\begin{array}{c}
\varepsilon_{x x e}^{(i) b} \\
\varepsilon_{y y e}^{(i) b} \\
\gamma_{x y e}^{(i) b}
\end{array}\right]=\mathbf{B}^{b} \mathbf{u}_{\varepsilon}^{(i) b},\left[\begin{array}{c}
\bar{\gamma}_{x z e}^{(i)} \\
\bar{\gamma}_{y z e}^{(i)}
\end{array}\right]=\mathbf{B}^{s} \mathbf{u}_{\varepsilon}^{(i) b},
$$


with

$$
\mathbf{B}^{b}=\left[\begin{array}{cccccc}
\mathbf{0} & \mathbf{0} & \mathbf{0} & \mathbf{0} & \mathbf{0} & z \mathbf{N} \\
\mathbf{0} & \mathbf{0} & -z \mathbf{N}_{, y} & \mathbf{0} & \mathbf{0} & \mathbf{0} \\
\mathbf{0} & \mathbf{0} & \mathbf{0} & -z \mathbf{N} & z \mathbf{N}_{y} & \mathbf{0}
\end{array}\right], \mathbf{B}^{s}=\left[\begin{array}{cccccc}
\mathbf{0} & \mathbf{N} & \mathbf{0} & \mathbf{0} & \mathbf{N} & \mathbf{0} \\
\mathbf{N}_{, y} & \mathbf{0} & -\mathbf{N} & \mathbf{0} & \mathbf{0} & \mathbf{0}
\end{array}\right]
$$

and

$$
\begin{gathered}
\multicolumn{10}{c}{\mathbf{u}_{\varepsilon}^{(i) b}=\boldsymbol{\aleph}_{\varepsilon e}^{(i) b} \mathbf{N}_{B \varepsilon}^{b} \Xi_{e}^{(i)}=} \\
=\left[\begin{array}{cccccc}
\mathbf{U}_{z e}^{(i)} & \mathbf{0} & \mathbf{0} & \mathbf{0} & \mathbf{0} & \mathbf{0} \\
\mathbf{0} & \mathbf{U}_{z e}^{(i)} & \mathbf{0} & \mathbf{0} & \mathbf{0} & \mathbf{0} \\
\mathbf{0} & \mathbf{0} & \Phi_{x e}^{(i)} & \mathbf{0} & \mathbf{0} & \mathbf{0} \\
\mathbf{0} & \mathbf{0} & \mathbf{0} & \Phi_{x e}^{(i)} & \mathbf{0} & \mathbf{0} \\
\mathbf{0} & \mathbf{0} & \mathbf{0} & \mathbf{0} & \Phi_{y e}^{(i)} & \mathbf{0} \\
\mathbf{0} & \mathbf{0} & \mathbf{0} & \mathbf{0} & \mathbf{0} & \Phi_{y e}^{(i)}
\end{array}\right]\left[\begin{array}{cc}
\mathbf{0} & \mathbf{N}_{B}\left(x_{e}\right) \\
\mathbf{0} & \mathbf{N}_{B}^{\prime}\left(x_{e}\right) \\
\mathbf{0} & \mathbf{N}_{B}\left(x_{e}\right) \\
\mathbf{0} & \mathbf{N}_{B}^{\prime}\left(x_{e}\right) \\
\mathbf{N}_{B}\left(x_{e}\right) & \mathbf{0} \\
\mathbf{N}_{B}^{\prime}\left(x_{e}\right) & \mathbf{0}
\end{array}\right]\left[\begin{array}{c}
\mathbf{A}_{i} \\
\mathbf{B}_{i}
\end{array}\right] .
\end{gathered}
$$

It has to be noted that $\mathbf{U}_{k e}^{(i)}$ and $\Phi_{k e}^{(i)}$ with $k=x, y, z$ refer to the corresponding nodal values for each SAFSE element $e$ extracted from the global warping and distortional mode couples $\mathbf{w}_{i}$ and $\mathbf{d}_{i}$ found in the cross-sectional analysis.

The displacement interpolations (27) and (30) are introduced in the virtual work of inertia terms

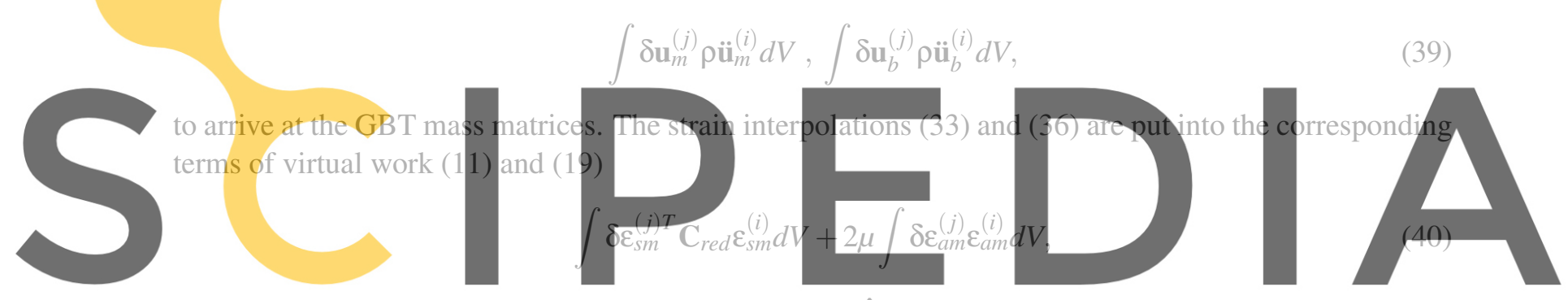

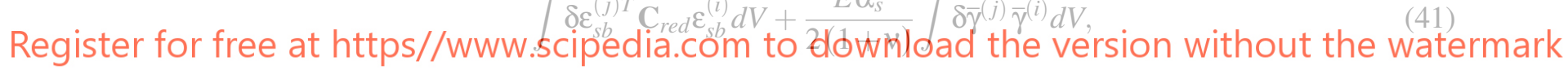

to find the GBT stiffness matrices, where $\mathbf{C}_{r e d}=\mathbf{C}\left(v^{*}=0\right)$ from (13). The resulting GBT formulation then reads,

$$
\mathbf{M}_{B} \ddot{\Xi}+\mathbf{K}_{B} \Xi=\mathbf{F}_{B},
$$

with $2 i_{\max }$ of generalized degrees of freedom at each node.

\section{Example}

For sake of the space only one example can be introduced here, where the performance of the proposed formulation can be shown. Consider a shaft of length $l=1 \mathrm{~m}$ with a homogeneous thin-walled $(s=$ $3 \mathrm{~mm}$ ) box-type cross-section of flange-width $w=0.1 \mathrm{~m}$ and web-height $h=0.04 \mathrm{~m}$ (profile center-lines are dimensioned, see Fig. 1). The left end $x=0$ is fully clamped and a harmonic torsional moment $\left(M_{0}=1 \mathrm{kNm}\right.$, excitation frequency $\left.\mathrm{v}\right)$ is applied onto a rigid mass-less load-sharing plate at $x=l$. Figure 1 shows the frequency response function (amplitude of torsion angle $\varphi_{x}$ at $x=l$ of steady state vibration with modal damping $\zeta=0.01$ ) evaluated by CBM (Vlasov's theory of torsion). A comparison to threedimensional continuum formulations and to an analysis using shell elements indicates the CBM results 

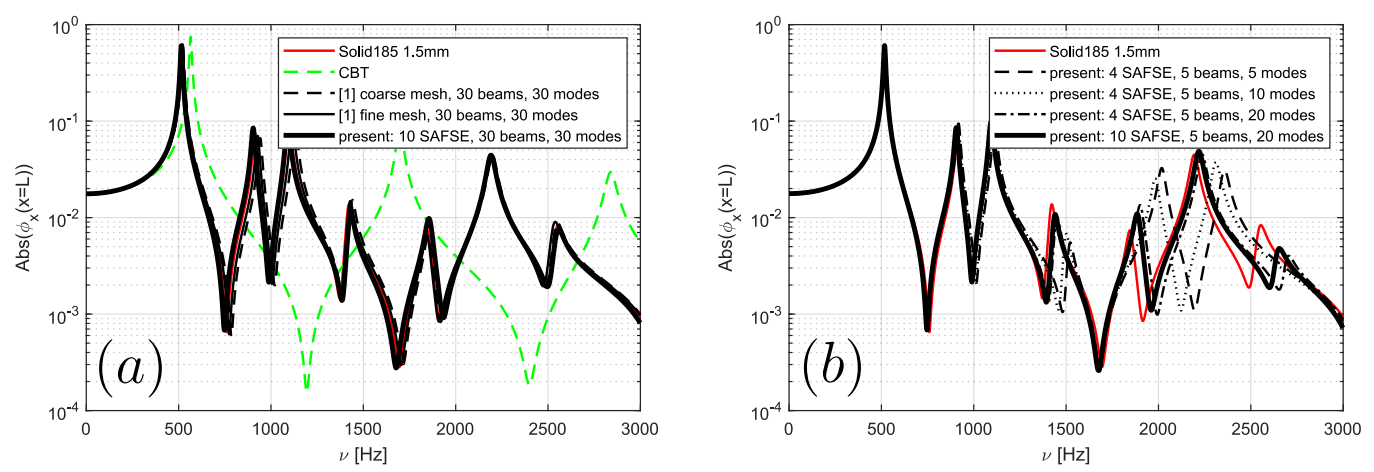

Figure 3: Frequency response function

do not even come close to reality (Fig. 1). The GBT procedure discussed in $[1,4]$ resolves those issues (see Fig. 3(a)), however, the applied two dimensional plane coarse mesh consists of $n=564$ nodes and 376 elements (two elements in wall-thickness direction), while the corresponding fine mesh has $n=2808$ nodes and 2340 elements (five elements in thickness direction). As a result, the cross-sectional analysis is based on a rather large eigenproblem with $3 n$ degrees of freedom. Figure 3(a) also indicates that the present procedure delivers the same accuracy compared to $[1,4]$, however, causes an eigenproblem with $6 n$ degrees of freedom with $n=30$ (ten SAFSEs). The coarsest discretization possible consists of only four elements with $n=12$ (flanges and webs of the cross-section are discretized with only one element). Figure 3(b) indicates that first three resonance spikes can be found accurately by using only five beam elements and $i_{\max }=5$ mode couples. Accuracy in higher frequency bands then requires more mode couples and more than one SAFSE in the flanges.

\section{Conclusion}

A generalized beam theory, where cross-sectional distortions and accompanied warping deformations can be introduced to overcome inaccuracies of classical beam mechanics, is typically a two-step algorithm consisting of a cross-sectional analysis and followed by a member analysis. The warping and distortional deformations of the cross-section are proposed to be extracted from a specific vibration analysis of a reference beam problem (cross-sectional analysis), and the resulting warping and distortion modes are then weighed and coupled axially in the member analysis. This fundamental strategy $[1,4]$ was shown to achieve high accuracy in problems where classical beam mechanics does not even come close to reality. In the present proceeding a line mesh formulation is proposed in the context of the GBT strategy discussed in [1,4]. We focus on cubic shape functions and derive membrane and bending properties independently. The membrane part consists of drilling rotations (mass-less micro-polar in-plane rotations) which are introduced based on an enhanced functional. In the bending part a shear elastic formulation is selected. The resulting SAFSE consists of six degrees of freedom at each node. The gain in efficiency compared to the procedures proposed in $[1,4]$ is overwhelming, and the high accuracy especially in beam dynamics with thin-walled box-type cross-sections remains.

Acknowledgments: The authors gratefully acknowledge financial support by the Slovak Grant Agency of the project VEGA No. 1/0102/18. 


\section{REFERENCES}

[1] S. Kugler, P. Fotiu, J. Murin, Analysis of Shells, Plates and Beams: A State of the Art Report, Springer, 2020, Ch. Beam Dynamics Using a Generalized Beam Theory Based on the Solution of a Reference Beam Problem.

[2] J. Murin, M. Aminbaghai, V. Kutis, Exact solution of the bending vibration problem of fgm beams with variation of material properties, Engineering Structures 32 (2010) 1631-1640.

[3] F. Gruttmann, W. Wagner, Shear correction factors in timoshenko's beam theory for arbitrary shaped cross-sections, Computational Mechanics 27 (2001) 199-207.

[4] S. Kugler, P. Fotiu, J. Murin, On the deficiencies of classical theories in predicting torsional frequencies of prismatic shafts, 14th International Conference on Vibration Problems, September 1-4 2019.

[5] E. Sapountzakis, A. Argyridi, Influence of in-plane deformation in higher order beam theories, Journal of Mechanical Engineering - Strojnicky Casopis 68 (3) (2018) 77-94.

[6] E. Sapountzakis, I. Dikaros, Advanced 3-d beam element including warping and distortional effects for the analysis of spatial framed structures, Engineering Structures 188 (2019) 147-164.

[7] O. C. Zienkiewicz, R. L. Taylor, Finite Element Method: Volume 2, Solid Mechanics (Finite Element Method), Butterworth-Heinemann, 2000.

[8] U. Schramm, L. Kitis, W. Kang, W. Pilkey, On the shear deformation coefficient in beam theory, Finite Elements in Analysis and Design 16 (1994) 141-162.

[9] W. Pilkey, W. Kang, U. Schramm, New structural matrices for a beam element with shear deformation, Finite Elements and Design 19 (1995) 25-44.

[10] D. Allman, A compatible triangular element including vertex rotations for plane elasticity analysis, Computers \& Structures 19 (1984) 1-8.

[11] T. J. Hughes, F. Brezzi, On drilling degrees of freedom, Computer Methods in Applied Mechanics and Engineering 72 (1989) 105-121.

[12] S. Kugler, P. Fotiu, J. Murin, A highly efficient membrane finite element with drilling degrees of freedom, Acta Mechanica 213 (2010) 323-348.

[13] S. Kugler, Development of a laterally pressed quadrilateral shell element, Ph.D. thesis, STU Bratislava (2010).

[14] S. Kugler, P. Fotiu, J. Murin, Advances in quadrilateral shell elements with drilling degrees of freedom, H. Altenbach and V. Eremeyev (eds.) Shell-like Structures - Advanced Structured Materials Vol. 15 (2011) 307-328.

[15] S. Kugler, P. Fotiu, J. Murin, The numerical analysis of fgm shells with enhanced finite elements, Engineering Structures 49 (2013) 920-935.

[16] S. Kugler, P. Fotiu, J. Murin, On consistent estimations of drilling rotations in shell elements, SSTA 2013 Danzig, Tenth Conference on Shell Structures - Theory and Applications. 